\title{
Amperometric Detection of Dopamine based on a Graphene Oxide/PEDOT:PSS Composite Electrode
}

\author{
Gilar Wisnu Hardi ${ }^{1}$, Siti Fauziyah Rahman ${ }^{1 *}$ \\ ${ }^{1}$ Biomedical Engineering, Department of Electrical Engineering, Faculty of Engineering, Universitas \\ Indonesia, Kampus UI Depok, Depok 16424, Indonesia
}

\begin{abstract}
Dopamine (DA) is a hormone and a neurotransmitter that plays many important roles within the brain and body. It is an organic compound in the catecholamines and phenethylamines groups. A considerable effort has been made since its discovery, and numerous techniques for DA detection have been developed. Graphene and its derivatives have great potential for the development of sensors and biosensors. Since it has excellent characteristics, such as good conductivity and a large surface area, a graphene-based biosensor is expected to have high sensitivity, selectivity, and long-term stability characteristics. Graphene oxide (GO) was synthesized using a chemical method through graphite oxidation. Graphene oxide/poly (3,4-ethylenedioxythiophene):poly (4-styrenesulfonate) (GO/PEDOT:PSS) composite films were prepared using an electropolymerization method on the surface of the working electrode. The properties of this composite electrode were characterized by cyclic voltammetry (CV) and scanning electron microscopy (SEM). The performance of the composite film was evaluated using three-electrode systems that consisted of a glassy carbon electrode (GCE) modifying a composite film electrode as a working electrode, a platinum electrode as an auxiliary electrode, and $\mathrm{Ag} / \mathrm{AgCl}$ as a reference electrode. The variation of the composite electrode was applied and evaluated to DA electrochemical sensing. The GO/PEDOT:PSS-modified electrode also exhibits high performance with a low detection limit of $1 \mu \mathrm{M}$. The results obtained have shown that GO/PEDOT:PSS/GCE composites are promising candidates for modifying electrode material used in electrochemical sensing.
\end{abstract}

Keywords: Biosensor; Dopamine; Electrochemical sensor; Electropolymerization; Graphene oxide; PEDOT:PSS

\section{Introduction}

Dopamine (DA) is one of the important neurotransmitters that plays a role in memory, hormonal, and cardiovascular processes (Sun et al., 2013; Zheng et al., 2015; Rahman, et al., 2016a). Neurological conditions like dementia, schizophrenia, and Parkinson's disease are likely to cause a deficiency or insufficient DA levels (Ali et al., 2007; Caudle et al., 2008; Guo et al., 2013). Various methods have been developed for the detection of DA. Biosensorbased electrochemical sensors are highly accurate, user friendly, and have a quick response time (Rahman, et al., 2016b; Hayat et al., 2019). Recently, chemically modified electrodes were established and reported with greater sensitivity and selectivity to effectively detect DA (Zhang et al., 2013; Wu et al., 2014; Xu et al., 2014). 
Graphene is widely used in electrochemical sensor production and holds great promise as an ideal candidate for sensing platforms. Materials based on graphene play an important role in every part of the environment (Arifutzzaman et al., 2019; Kusrini et al., 2019). Graphene is one of the greatest of recently studied materials, particularly in the field of electronics. It has many applications because of its characteristics, such as being the thinnest, strongest, and most conductive material (Morozov et al., 2008). Graphenes can be effectively used in electrochemical sensing systems for the selective detection of chemical species. One of the methods to synthesize graphene oxide (GO) through chemical synthesis, known as the Hummers method, is achieved by adding an oxidizing agent into the concentrated acid that contains graphite. Many researchers modify the methods to synthesize the graphene (Hummers and Offeman, 1958). One famous modification was carried out by Marcano et al. and is known as the Tour method. The Tour method demonstrates a less risky method since it does not use sodium nitrate $\left(\mathrm{NaNO}_{3}\right)$ and is a more effective graphite oxidation mechanism (Marcano et al., 2010).

The present research aimed to synthesize graphene oxide and investigate electrochemical studies of GO with poly (3,4-ethylene-dioxythiophene):poly (4styrenesulfonate) (PEDOT:PSS) polymer on the surface of a glassy carbon electrode (GCE). GO was chosen since it is cheaper and easier to spread into a homogeneous PEDOT: PSS solution compared to reduced graphene. The properties of GO are hydrophilic and PEDOT: PSS is soluble in water. The modified electrodes used to study DA oxidation are provided by cyclic voltammetry (CV).

\section{Methods}

\subsection{Chemicals}

Graphite flakes (99\% carbon basis, -325 mesh particle size), dopamine hydrochloride, and PEDOT:PSS were purchased from Sigma Aldrich, Singapore. Ethanol (99\%), sulfuric acid $\left(\mathrm{H}_{2} \mathrm{SO}_{4}\right)$, orthophosphoric acid $\left(\mathrm{H}_{3} \mathrm{PO}_{4}\right)$, potassium permanganate $\left(\mathrm{KMnO}_{4}\right), 30 \% \mathrm{H}_{2} \mathrm{O}_{2}$, and hydrochloric acid ( $\mathrm{HCl})$ 37\% were purchased from Mercks, Germany. Alumina polish with $0.05 \mu \mathrm{m}$ particle size was obtained from Basinc, USA. The supporting electrolyte, sodium phosphate buffer solution, was prepared by using disodium hydrogen phosphate $\left(\mathrm{Na}_{2} \mathrm{HPO}_{4}\right)$ and monosodium phosphate $\left(\mathrm{NaH}_{2} \mathrm{PO}_{4}\right)$ solutions in distilled water. All chemicals used for this work were analytical grade and all the solutions were prepared using distilled water without any further purification.

\subsection{Apparatus}

CV was performed with a Rodeostat electrochemical analyzer open source potentiostat (IO Rodeo, USA) with Sigma Plot software. The three electrodes used for electrochemical experiments consisted of $\mathrm{Ag} / \mathrm{AgCl}$ as reference electrodes, platinum auxiliary electrodes, and modified GCE working electrodes. All the electrodes were purchased from Basinc, USA. All measurements were carried out at room temperature.

\subsection{Graphene Oxide Synthesis using the Tour Method}

The GO was synthesized using the Tour method (Marcano et al., 2010) by mixing $90 \mathrm{~mL}$ of concentrated $\mathrm{H}_{2} \mathrm{SO}_{4}$ and $10 \mathrm{~mL}$ of concentrated $\mathrm{H}_{3} \mathrm{PO}_{4}$ at a ratio of 9:1. Orthophosphoric acid was added to avoid further oxidation (Higginbotham et al., 2010). First, $0.5 \mathrm{~g}$ of graphite powder was poured into the concentrated acid. Then $4.5 \mathrm{~g}$ of $\mathrm{KMnO}_{4}$ was poured slowly into the mixture. The temperature was then changed from $40^{\circ} \mathrm{C}$ to $50^{\circ} \mathrm{C}$, and the mixture was then stirred for 12 hours.

The mixture transforms into a paste as the reaction progresses. The mixture was cooled to room temperature. The mixture was then added to $250 \mathrm{ml}$ of distilled water to stop the 
reaction. Ten milliliters of $30 \% \mathrm{H}_{2} \mathrm{O}_{2}$ was used to reduce the manganese ions and to dissolve the manganese sulfate and manganese oxide. $\mathrm{H}_{2} \mathrm{O}_{2}$ aims to reduce the remaining $\mathrm{KMnO}_{4}$ in acidic media and dissolved manganese sulfate. Bubbling occurred when $30 \%$ of $\mathrm{H}_{2} \mathrm{O}_{2}$ was applied, and a bright yellow color appeared, suggesting that high oxidation had occurred. The mixture was filtered to separate between cake and solution using filter paper. The cake was washed with $5 \% \mathrm{HCl}$. The cake was then washed two times using water and alcohol by centrifugation. The resulting supernatant had been decanted away. The cake that was collected was stirred for 12 hours in distilled water at $60^{\circ} \mathrm{C}$. This method is called exfoliation (Emiru and Ayele, 2017). After that, the graphene oxide was dried for 24 hours at $70^{\circ} \mathrm{C}$.

\subsection{Fabrication of a Working Electrode}

First, the GCE electrode was sequentially polished with alumina slurry to create a mirror-like surface. It was then rinsed and cleaned using ultrasonic equipment for 3 minutes in water and ethanol, 1:1, and dried at room temperature. Second, the suspension of GO/PEDOT: PSS ( $8 \mu \mathrm{L}$ ) was cast onto the GCE surface and kept at room temperature for 1 hour to ensure that it was dry, smooth, and stable. Third, the electropolymerization of GCE using GO (0.01 g), PEDOT:PSS, and a phosphate buffer was conducted by CV. CV was performed from -1.4 to $+2.4 \mathrm{~V}$ for ten cycles with a scan rate of $100 \mathrm{mV} \mathrm{s}^{-1}$. Before being used, the modified electrode was again electropolymerized with $\mathrm{CV}$ from +0.3 to $+1.2 \mathrm{~V}$ in a $0.1 \mathrm{M}$ phosphate buffer solution of $\mathrm{pH} 7$ until it was a stable cyclic voltammogram (Xu et al., 2010).

\subsection{Electrochemical Measurements}

Electrochemical measurements were performed using an open-source potentiostat (IO Rodeo, USA). The electrochemical cells containing the modified GCE ( $3 \mathrm{~mm}$ diameter) as the working electrode, the platinum wire as the counter, and the $\mathrm{Ag} / \mathrm{AgCl}$ as the reference electrode were connected to the potentiostat, as shown in Figure 1. All experiments were performed at room temperature.

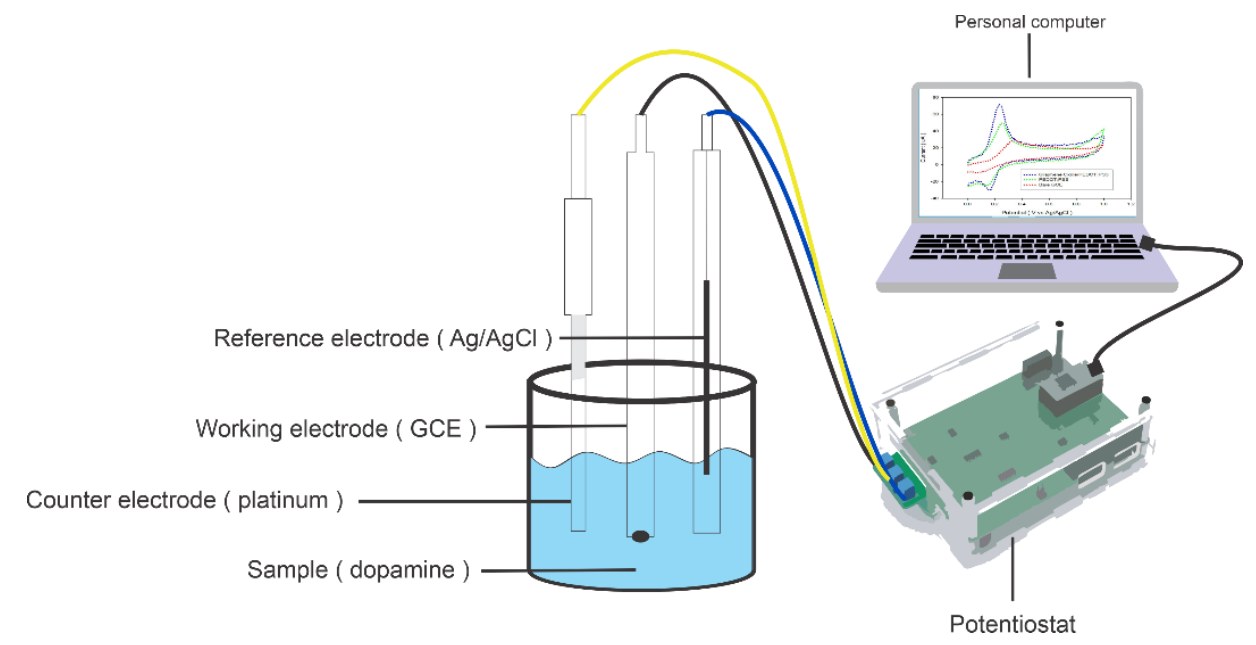

Figure 1 Setup CV measurement

\section{Results and Discussion}

\subsection{Visual Observation}

Figure $2 \mathrm{a}$ shows the graphite oxidized by $\mathrm{H}_{2} \mathrm{SO}_{4} / \mathrm{H}_{3} \mathrm{PO}_{4}$ and $\mathrm{KMnO}_{4}$ with a dark greenish color. The oxidation cycle is improved by combining sulfuric acid, orthophosphoric acid, and potassium permanganate (Marcano et al., 2010). The paste has been 
mixed with $250 \mathrm{~mL}$ of water and $10 \mathrm{~mL}$ of $30 \% \mathrm{H}_{2} \mathrm{O}_{2}$ resulting in a brownish-yellow suspension, as shown in Figure 2b. This full-color switch suggested a greater degree of graphite oxidation to GO. The model in Figure 2c shows that the GO suspension is filtered directly with acids and metal ions, and then the filter surface is easily covered by a compact film, as shown in Figure 2d. The suspension was centrifuged (Figure 2e) with the elimination of the supernatant. Figure $2 \mathrm{e}$ shows a dark brown paste. The cake was exfoliated for 12 hours at $60^{\circ} \mathrm{C}$, as shown in Figure 2f. Eventually, the GO solution turns black. The resulting GO was then filtered and dried in an oven (Figures $2 \mathrm{~g}$ and $2 \mathrm{~h}$ ).

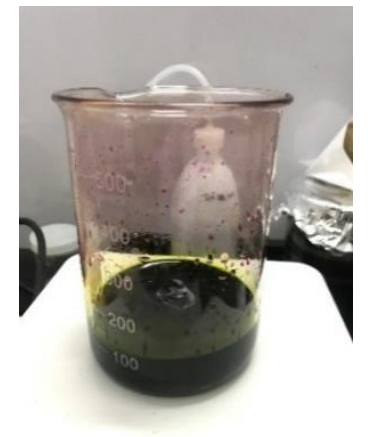

(a)

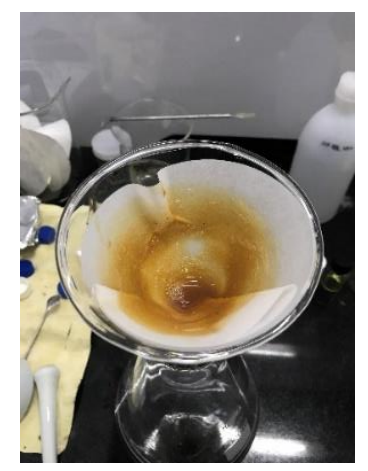

(d)

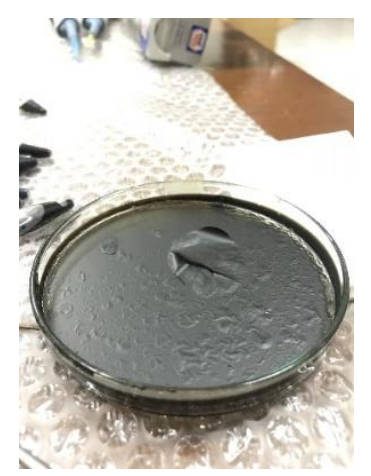

(g)

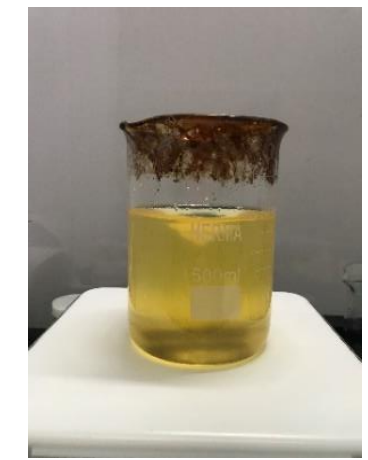

(b)

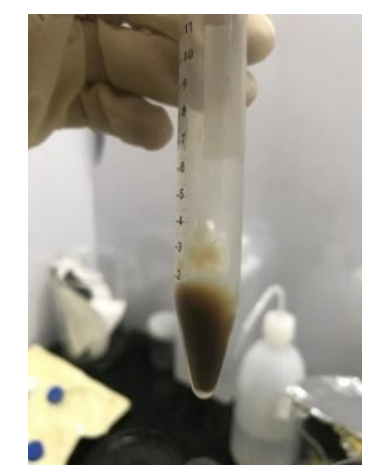

(e)

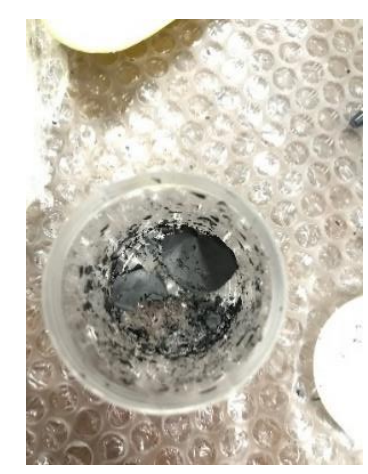

(h)

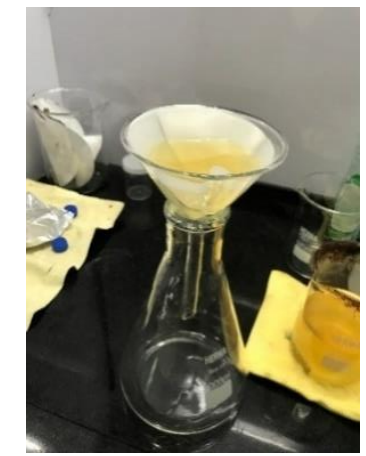

(c)

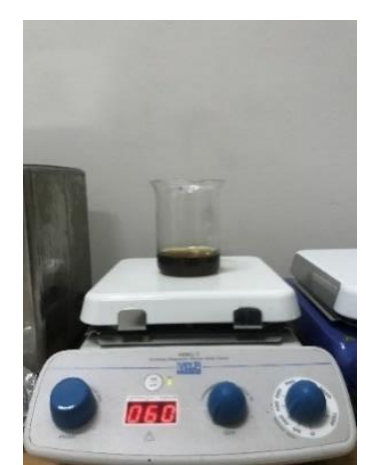

(f)

Figure 2 Synthesis of graphene oxide

Figure 3 shows the graphene oxide synthesis mechanism using the Tour method. Two major important steps in graphene synthesis take place in the graphite layer, namely oxidation and exfoliation. The first step in the GO synthesis mechanism begins after graphite has been mixed into $\mathrm{H}_{2} \mathrm{SO}_{4}$ and $\mathrm{H}_{3} \mathrm{PO}_{4}$ as an intercalant. As a result of this mechanism, graphite layers tend to separate. The oxidizing stage happens after $\mathrm{KMnO}_{4}$ as 
an oxidizing agent was added into the solution. The oxidizing stage converts graphite powder to oxidized graphite, which is known as pristine oxide of graphite (PGO). The next step is to convert PGO to GO after water has been added, which involves sulfate hydrolysis and the release of the interlayer PGO.

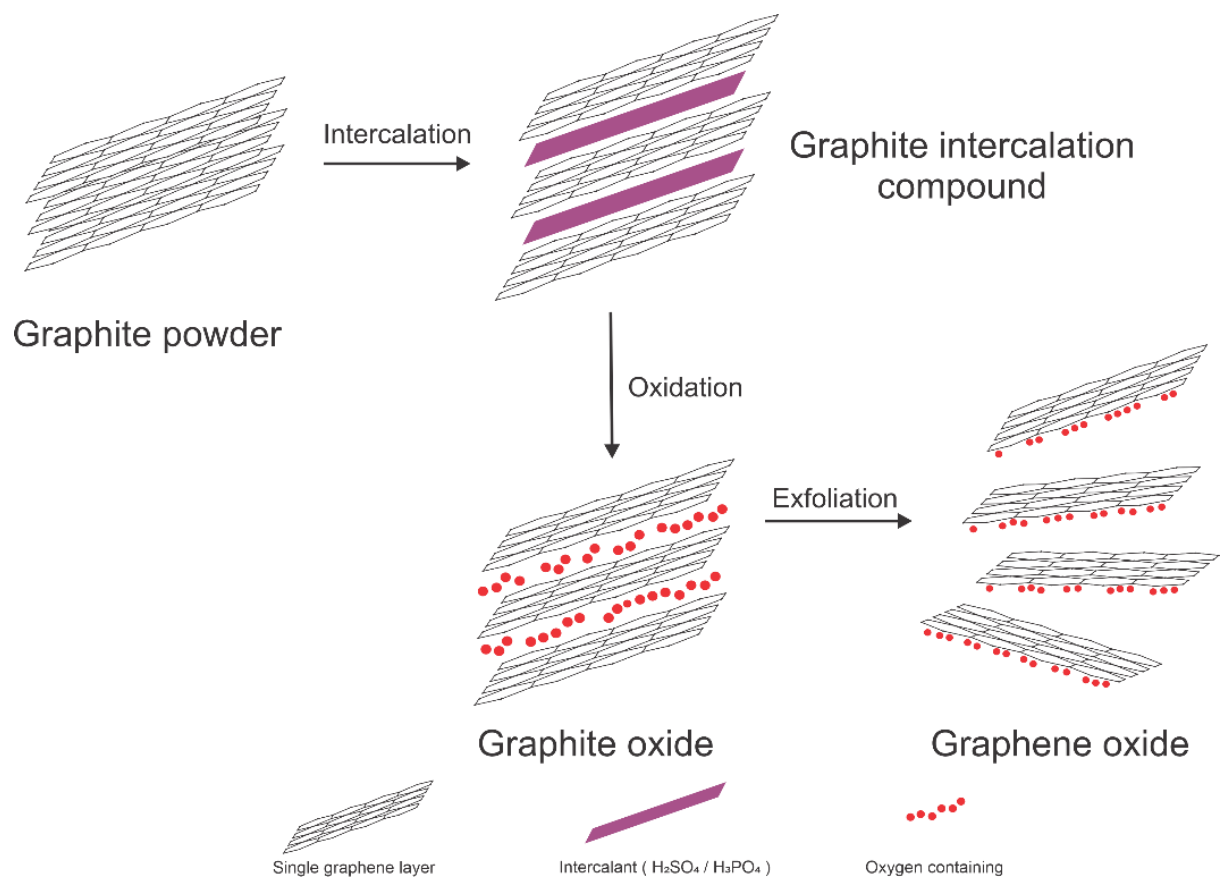

Figure 3 The graphene oxide synthesis mechanism

Figure 4 shows the scanning electron microscopy (SEM) images of the GO. The sample shows platelike forms. GO morphology is observed as a flaccid texture which represents its layered microstructure, as shown in Figure 4. Figure 4 clearly indicates the wider interspaces of the sheet and the thinner edges of the GO paper. The SEM images of graphene were similar to other researchers (Marcano et al., 2010; Paulchamy et al., 2015), which indicates that graphene oxide was formed through this mechanism.

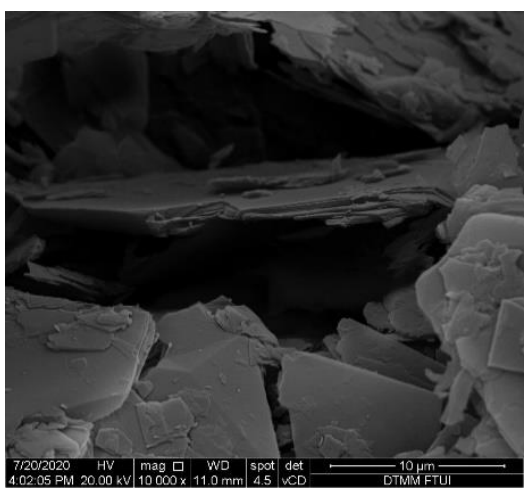

(a)

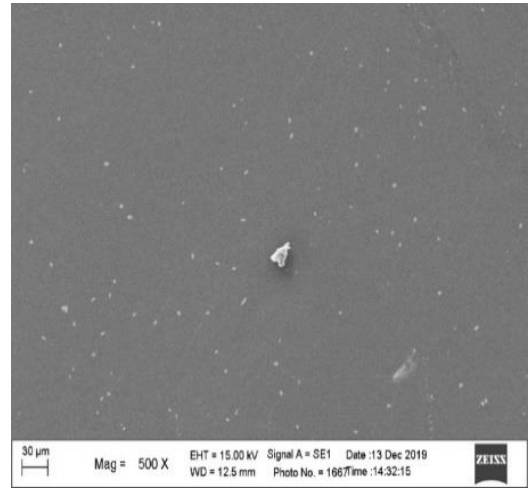

(b)

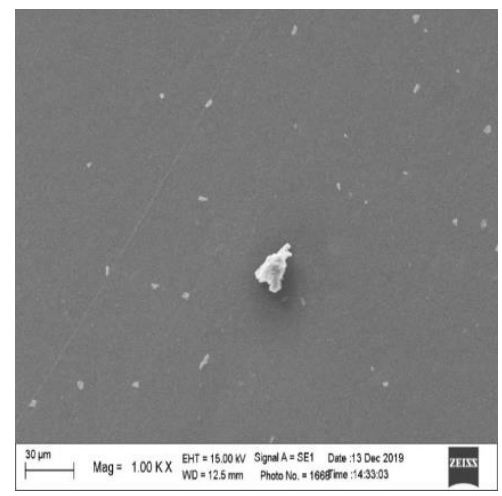

(c)

Figure 4 SEM images of graphite (a), graphene oxide with $500 \times($ b) and $1000 \times$ magnification (c)

\subsection{Electropolymerization Characteristics of the Modified Electrode}

The electropolymerization modified electrode using polymer (in this research using PEDOT: PSS) obtains stable, uniform, and compact polymer films on the electrode surface 
(Wang and Hui, 2017). Figure 5 shows the CV electropolymerization characteristics of GO/PEDOT:PSS on the surface of the GCE as a working electrode. Two anodic peaks and one cathodic peak were formed during the electropolymerization process. The anodic peaks were approximately $0.321 \mathrm{~V}$ and $1.636 \mathrm{~V}$. The cathodic peak was approximately $-0.479 \mathrm{~V}$. The anodic and cathodic peak currents steadily improved as the scanning cycles improved, and the resulting potential changed to more positive and negative values, respectively. These findings revealed that the polymer film had accumulated on the electrode surface, and with increasing scanning cycles, its thickness grew steadily.

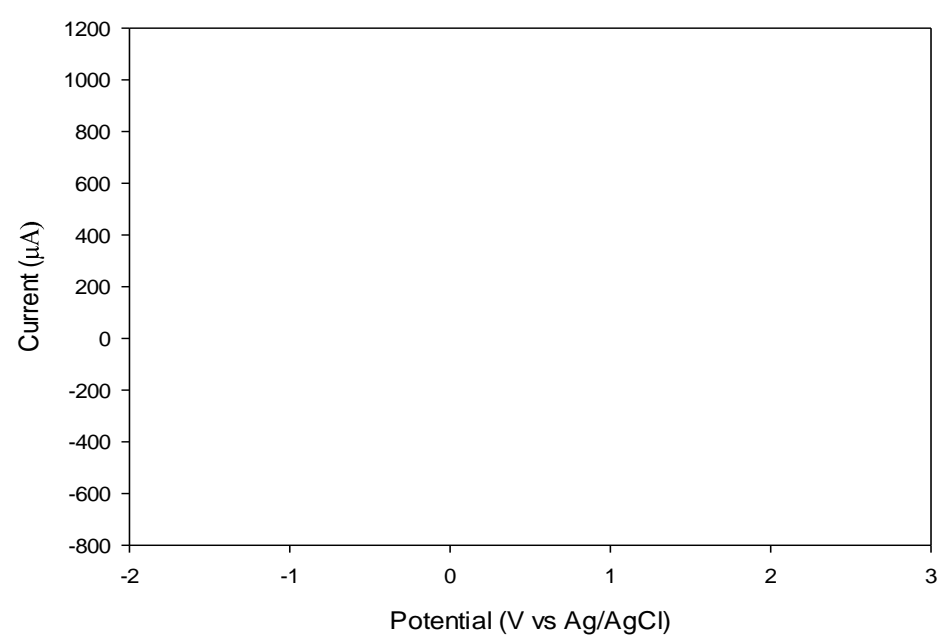

Figure 5 Cyclic voltammograms of electropolymerization GO/PEDOT: PSS-modified GCE. Scan rate: $100 \mathrm{mV} \mathrm{s}-1$; ten cycles

\subsection{Electrochemical Characteristics of DA on Modified Electrode}

Dopamine (DA) detection was achieved using CV. The electro-oxidation of DA was first investigated by $\mathrm{CV}$ in a phosphate buffer at pH 7 in bare and modified GCE (Figure 6). PEDOT:PSS-modified GCE was observed with a pair of distinct redox peaks of DA, suggesting a reversible electron transfer cycle. But the $\mathrm{CV}$ response of $\mathrm{DA}$ to GO/PEDOT:PSS-modified GCE is larger than that of PEDOT:PSS-modified GCE and bare GCE, which is attributed to high electrical conductivity and a large specific surface area of GO. Therefore, these results suggest that the GO and PEDOT:PSS materials were successfully modified on the GCE surface and could accelerate the movement of electrons.

As shown in Figure 6, the electrochemical behaviors of DA in different electrodes such as bare GCE, PEDOT:PSS/GCE, and GO/PEDOT:PSS/GCE were investigated using a CV method containing $1 \mathrm{mM}$ DA. In the presence of DA, GO/PEDOT:PSS-modified GCE exhibits the highest cathodic peak currents. The result was similar to Gong et al. (2020). This sensor shows a higher oxidation peak of DA with graphene modification. The difference in peak potential $(\triangle \mathrm{Ep})$ between the anodized peak (Epa) and the cathodic peak (Epc) in PEDOT:PSS-modified GCE was calculated as $0.11 \mathrm{~V}$. The peak current of DA with GO/PEDOT:PSS-modified GCE corresponding to $\triangle \mathrm{Ep}$ was calculated as $0.08 \mathrm{~V}$, which indicates that the electrochemical reaction process of DA was fast and reversible. Meanwhile, GO/PEDOT:PSS-modified GCE possessed a much higher peak oxidation current and more negative peak oxidation potential of DA, indicating good electrocatalytic oxidation capability of DA. Compared to bare GCE and PEDOT:PSS-modified GCE, the GO/PEDOT:PSS-modified GCE had a high electrocatalytic activity and good conductivity. 




Figure 6 Cyclic voltammogram of $1 \mathrm{mM}$ dopamine, with bare GCE, PEDOT:PSS-modified GCE, and GO/PEDOT: PSS-modified GCE at pH 7 in a $0.1 \mathrm{M}$ phosphate buffer at $\mathrm{pH} 7.0$. Scan rate: $0.05 \mathrm{~V}$

\subsection{Performance of GO/PEDOT:PSS-Modified GCE}

We evaluated the performance of the amperometric biosensor of DA, such as sensitivity, linear detection range, and limit of detection by cyclic voltammogram to validate the relationship between the oxidation peak current and DA concentration. The oxidation peak of DA was linearly dependent on DA concentration in the range of $0.001-1.0 \mathrm{mM}$ (Figure 7).

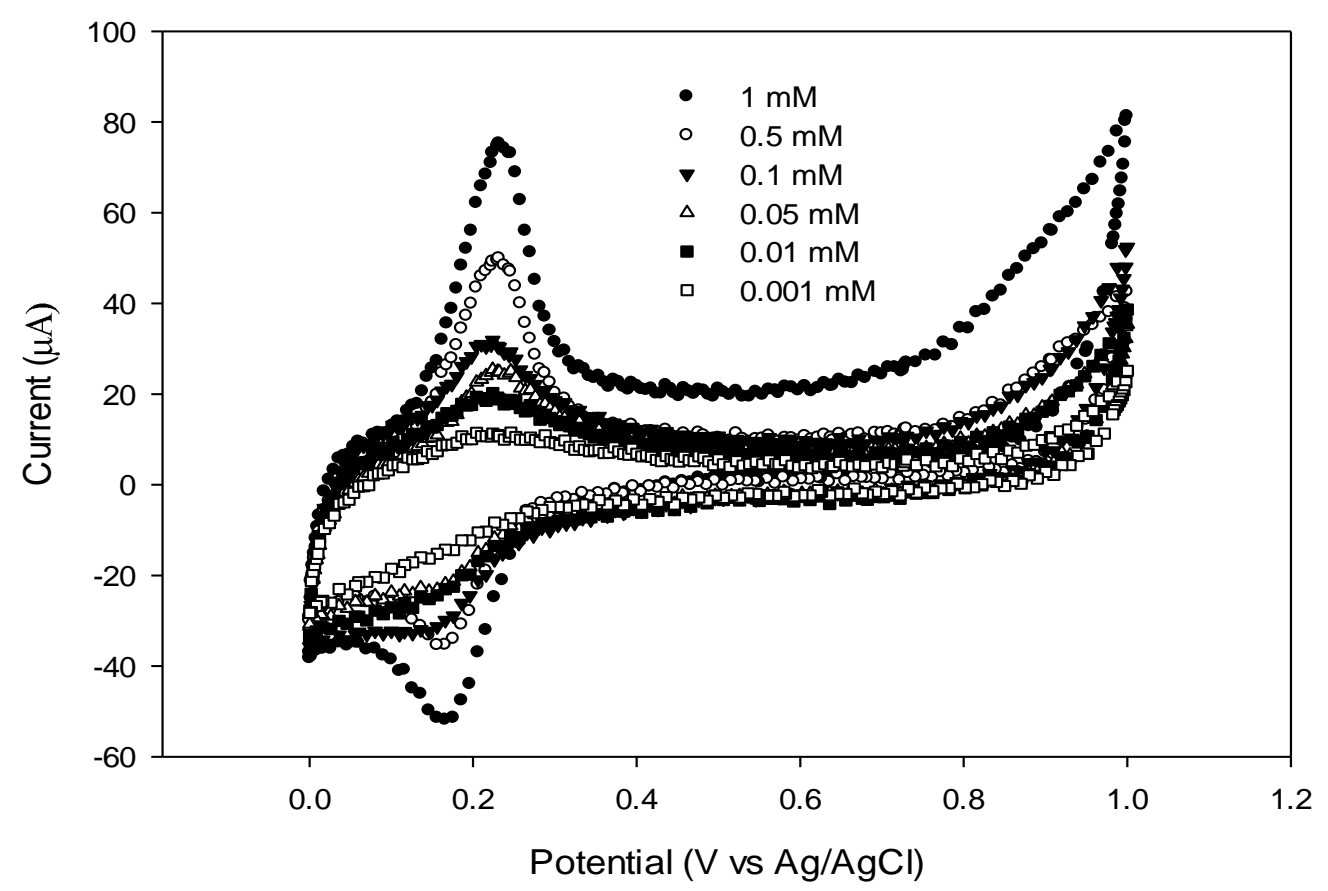

Figure 7 Amperometric response of GO/PEDOT:PSS-modified GCE to the various concentrations of DA with a scan rate of $0.05 \mathrm{~V}$. The inset shows linearity between the concentration of DA and the anodic current response 
The inset of Figure 7 shows the regression equation for DA oxidation. The linear regression equation can be expressed as $\mathrm{I}_{\mathrm{pa}}(\mu \mathrm{A})=58.824$ Conc $_{\text {dopamine }}(\mathrm{mM})+18.071\left(\mathrm{R}^{2}=0.9833\right)$. Consequently, the sensitivity and linear detection range of the electropolymerization of G0/PEDOT:PSS-modified GCE was 1-1,000 $\mu \mathrm{M}$. The limit of detection was estimated to be $1 \mu \mathrm{A}$. Similar to our research, Rahman et al. modified the surface of the electrode using a multi-walled carbon nanotube (MWNT) and tyrosinase enzyme. Tyrosinase/MWNT/GCE exhibits a detection limit of $50 \mu \mathrm{A}$ (Rahman et al., 2016b). Aravind et al. fabricated $\mathrm{Au} / \mathrm{TiO}_{2}$ nanotube films on GCE which resulted in a detection limit of $3 \mu \mathrm{M}$ (Aravind et al., 2011).

\section{Conclusions}

The graphene oxide synthesis was achieved by mixing graphite powder into a concentrated acid medium with the existence of an oxidizing agent. Graphite oxidation using the Tour method is more efficient and less hazardous than other methods because it is cost-effective, non-toxic, and environmentally friendly. There are two major important steps to the synthesis mechanism of graphene oxide, namely oxidation and exfoliation.

The modified GCE by GO/PEDOT: PSS film exhibits high electrocatalytic behavior for DA oxidation. The electropolymerization of GO/PEDOT: PSS on the surface of the electrode exhibits a detection limit of $1 \mu \mathrm{A}$ and a wide linear range $(1-1,000 \mu \mathrm{M})$. This composite electrode was used for rapid-current response DA detection, which offered a promising method for modified electrode materials.

\section{Acknowledgements}

We gratefully acknowledge the funding from Universitas Indonesia through Publikasi Terindeks Internasional (PUTI) Q1 2020 No. NKB-1422/UN2.RST/HKP.05.00/2020. We express our gratitude to Dr. Eng. Arief Udhiarto, S.T., M.T., Head of Laboratory Nanodevice, MRPQ building for the use of his laboratory facilities during this research.

\section{References}

Ali, S.R., Ma, Y., Parajuli, R.R., Balogun, Y., Lai, W.Y.C., He, H., 2007. A Nonoxidative Sensor based on a Self-doped Polyaniline/Carbon Nanotube Composite for Sensitive and Selective Detection of the Neurotransmitter Dopamine. Analytical Chemistry, Volume 79(6), pp. 2583-2587

Aravind, S.S.J., Srinivasan, S.K., Ramaprabhu, S., 2011. Au/TiO 2 Nanotubes for Selective Detection of Dopamine. International Journal of Nanoscience, Volume 10(4-5), pp. 1185-1189

Arifutzzaman, A., Ismail, A.F., Alam, M.Z., Khan, A.A., Saidur, R., 2019. Investigation of Extraction Yields of Exfoliated Graphene in Deionized Water from Organic Solvents. International Journal of Technology, Volume 10(6), pp. 1251-1259

Caudle, W.M., Colebrooke, R.E., Emson, P.C., Miller, G.W., 2008. Altered Vesicular Dopamine Storage in Parkinson's Disease: A Premature Demise. Trends in Neurosciences, Volume 31(6), pp. 303-308

Emiru, T.F., Ayele, D.W., 2017. Controlled Synthesis, Characterization and Reduction of Graphene Oxide: A Convenient Method for Large Scale Production. Egyptian Journal of Basic and Applied Sciences, Volume 4(1), pp. 74-79

Gong, Q.J., Han, H.X., Wang, Y.D., Yao, C.Z., Yang, H.Y., Qiao, J.L., 2020. An Electrochemical Sensor for Dopamine Detection using Poly-Tryptophan Composited Graphene on Glassy Carbon as the Electrode. Xinxing Tan Cailiao/New Carbon Materials, Volume 35(1), pp. 34-41 
Guo, Z., Seol, M.L., Kim, M.S., Ahn, J.H., Choi, Y.K., Liu, J.H., Huang, X.J., 2013. Sensitive and Selective Electrochemical Detection of Dopamine using an Electrode Modified with Carboxylated Carbonaceous Spheres. Analyst, Volume 138(9), pp. 2683-2690

Hayat, Moh., Saepudin, E., Einaga, Y., Ivandini, T.A., 2019. CdS Nanoparticle-based Biosensor Development for Aflatoxin Determination. International Journal of Technology, Volume 10(4), pp. 787-797

Higginbotham, A.L., Kosynkin, D.V., Sinitskii, A., Sun, Z., Tour, J.M., 2010. Lower-Defect Graphene Oxide Nanotubes. ACS Nano, Volume 4(4), pp. 2059-2069

Hummers, W.S., Offeman, R.E., 1958. Preparation of Graphitic Oxide. Journal of the American Chemical Society, Volume 80(6), p. 1339

Kusrini, E., Suhrowati, A., Usman, A., Khalil, M., Degirmenci, V., 2019. Synthesis and Characterization of Graphite Oxide, Graphene Oxide, and Reduced Graphene Oxide from Graphite Waste using Modified Hummers' Method and Zinc as Reducing Agent. International Journal of Technology, Volume 10(6), pp. 1093-1104

Marcano, D.C., Kosynkin, D.V., Berlin, J.M., Sinitskii, A., Sun, Z., Slesarev, A., Alemany, L.B., Lu, W., Tour, J.M., 2010. Improved Synthesis of Graphene Oxide. ACS Nano, Volume 4(8), pp. 4806-4814

Morozov, S.V., Novoselov, K.S., Katsnelson, M.I., Schedin, F., Elias, D.C., Jaszczak, J.A., Geim, A.K., 2008. Giant Intrinsic Carrier Mobilities in Graphene and Its Bilayer. Physical Review Letters, Volume 100(1), pp. 11-14

Paulchamy, B., Arthi, G., Lignesh, B., 2015. A Simple Approach to Stepwise Synthesis of Graphene Oxide Nanomaterial. Journal of Nanomedicine \& Nanotechnology, Volume 06(01), pp. 1-4

Rahman, S.F., Gobikhrisnan, S., Gozan, M., Jong, G.T., Park, D.H., 2016a. L-DOPA Synthesis using Tyrosinase-Immobilized on Electrode Surfaces. Korean Chemical Engineering Research, Volume 54(6), pp. 817-821

Rahman, S.F., Min, K., Park, S.H., Park, J.H., Yoo, J.C., Park, D.H., 2016b. Highly Sensitive and Selective Dopamine Detection by an Amperometric Biosensor based on Tyrosinase/MWNT/GCE. Korean Journal of Chemical Engineering, Volume 33(12), pp. 3442-3447

Sun, W., Wang, X., Wang, Y., Ju, X., Xu, L., Li, G., Sun, Z., 2013. Application of Graphene-SnO 2 Nanocomposite Modified Electrode for the Sensitive Electrochemical Detection of Dopamine. Electrochimica Acta, Volume 87, pp. 317-322

Wang, J., Hui, N., 2017. A Nanocomposite Consisting of Flower-Like Cobalt Nanostructures, Graphene Oxide and Polypyrrole for Amperometric Sensing of Nitrite. Microchimica Acta, Volume 184(7), pp. 2411-2418

Wu, D., Li, Y., Zhang, Y., Wang, P., Wei, Q., Du, B., 2014. Sensitive Electrochemical Sensor for Simultaneous Determination of Dopamine, Ascorbic Acid, and Uric Acid Enhanced by Amino-Group Functionalized Mesoporous $\mathrm{Fe}_{3} \mathrm{O}_{4} @ G r a p h e n e$ Sheets. Electrochimica Acta, Volume 116, pp. 244-249

Xu, T.Q., Zhang, Q.L., Zheng, J.N., Lv, Z.Y., Wei, J., Wang, A.J., Feng, J.J., 2014. Simultaneous Determination of Dopamine and Uric Acid in the Presence of Ascorbic Acid using Pt Nanoparticles Supported on Reduced Graphene Oxide. Electrochimica Acta, Volume 115, pp. 109-115

Xu, X.L., Huang, F., Zhou, G.L., Zhang, S., Kong, J.L., 2010. A Novel Electrochemical Sensor for Probing Doxepin Created on a Glassy Carbon Electrode Modified with Poly(4-AminoBenzoic Acid)/Multi-Walled Carbon Nanotubes Composite Film. Sensors, Volume 10(9), pp. 8398-8410 
Zhang, B., Huang, D., Xu, X., Alemu, G., Zhang, Y., Zhan, F., Shen, Y., Wang, M., 2013. Simultaneous Electrochemical Determination of Ascorbic Acid, Dopamine and Uric Acid with Helical Carbon Nanotubes. Electrochimica Acta, Volume 91, pp. 261-266

Zheng, X., Guo, Y., Zheng, J., Zhou, X., Li, Q., Lin, R., 2015. Simultaneous Determination of Ascorbic Acid, Dopamine and Uric Acid using Poly(L-Leucine)/DNA Composite Film Modified Electrode. Sensors and Actuators, B: Chemical, Volume 213, pp. 188-194 